\title{
APORTES PARA UNA PEDAGOGÍA CRITICA NUESTROAMERICANA: IDENTIFICANDO EL NUUCLEO CONCEPTUAL DEL PENSAMIENTO POLÍTICO-PEDAGOGGICO DE PAULO FREIRE
}

\author{
CONTRIBUTIONS TO A LATIN AMERICAN CRITICAL PEDAGOGY: \\ IDENTIFYING THE CONCEPTUAL CONCEPT OF PAULO FREIRE'S \\ POLITICAL-PEDAGOGICAL THOUGHT \\ CONTRIBUIÇÕES PARA UMA PEDAGOGIA CRÍTICA LATINO-AMERICANA: \\ IDENTIFICANDO O NÚCLEO CONCEITUAL DO PENSAMENTO POLÍTICO- \\ PEDAGÓGICO DE PAULO FREIRE
}

Jorge Alejandro Santos* jorgesantosuba@gmail.com

\begin{abstract}
REVISTA PEDAGÓGICA
Revista do Programa de Pós-graduação em Educação da Unochapecó | ISSN 1984-1566

Universidade Comunitária da Região de Chapecó | Chapecó-SC, Brasil

Como referenciar este artigo: SANTOS, J. A. Aportes para una pedagogía crítica nuestroamericana: identificando el núcleo conceptual del pensamiento político-pedagógico de Paulo Freire. Revista Pedagógica, Chapecó, v. 19, n. 41, p. 80-95, maio./ago. DOI: http://dx.doi.org/10.22196/rp.v19i41.3801
\end{abstract}

RESUMEN: El artículo tiene como objetivo rastrear, en la producción teórica y en la práctica pedagógica de Paulo Freire, los puntos nodales de su pensamiento que, más allá de los posicionamientos estratégicos y las reelavoraciones teóricas relativas al contexto y a los escenarios políticos en disputa, están presentes en toda su obra. Estos principios nos sirve hoy para repensar los desafíos de la educación Latinoamericana y para postularlos como base de cualquier sistema educativo que busque construir una región más justa, libre, democrática y soberana. A fin de alcanzar ese objetivo el trabajo comenzará planteando los puntos que hacen complejo el abordaje del pensamiento de Freire. Luego propondrá una clasificación temporal y conceptual a fin de ordenar por etapas los distintos giros en la perspectiva del autor y, finalmente, identificará los puntos centrales que recorren todas las etapas y estructuran sus desarrollos arribando al núcleo de la reflexión freireana y a su legado más fecundo.

Palabras clave: Educación. Pedagogía. Liberación. Oprimidos. Freire.

ABSTRACT: The article aims to trace, in the theoretical
production and pedagogical practice of Paulo Freire, the
nodal points of his thought that, beyond the strategic
positions and theoretical reelavorations related to the
context and political scenarios in dispute, are present In
all his work. These principles serve us today to rethink the
challenges of Latin American education and to postulate
them as the basis of any educational system that seeks
to build a more just, free, democratic and sovereign
region. In order to reach this goal the work will begin by raising the points that make the approach of Freire's thinking complex. Then he will propose a temporal and conceptual classification in order to order in stages the different turns in the perspective of the author and finally identify the central points that run through all the stages and structure their development arriving at the core of Freirean reflection and his most fruitful legacy.

Keywords: Education. Pedagogy. Liberation. Oppressed. Freire.

RESUMO: O artigo tem como objetivo pesquisar a produção teórica e prática pedagógica de Paulo Freire, para encontrar os pontos nodais de seu pensamento que, além de posicionamentos estratégicos e reelavoraciones teóricas sobre o contexto e cenários políticos em disputa, estão presentes em toda a sua obra. Estes princípios são úteis hoje para repensar os desafios da educação latino-americana e postular-los como base para qualquer sistema educacional que busca construir uma sociedade mais justa, uma região mais livre, democrática e soberana. Para alcançar este objectivo, o trabalho levanta pontos que tornam complexa abordar o pensamento de Freire. Em seguida, propor uma classificação temporária e estágios conceituais para classificar as várias mudanças na perspectiva do autor e, finalmente, identificar os pontos-chave que estruturam o seu desenvolvimento. Esperamos chegar ao núcleo da reflexão de Freire e seu legado mais fértil.

Palavras-chave: Educação. Pedagogia. Libertação. Oprimido. Freire 
* Abogado, Licenciado en Filosofía y Doctor en Filosofía por la Universidad de Buenos Aires (2009-2015-UBA). Actualmente posdoctorando por la Unochapecó. Profesor de Pos-graduación en Filosofía, Facultad de Filosofía y Letras UBA. Profesor de Filosofía de la Ciencia en el Ciclo Básico Común UBAy en la Facultad de Ciencias Económicas de la Universidad de Lomas de Zamora. Principal foco de actuación: Filosofía de la Educación (perspectiva crítica y liberadora), Filosofía Intercultural y Educación Intercultural (formación de educadores interculturales y educación intercultural escolar). Investigador del Instituto de Filosofía de la Facultad de Filosofía y Letras UBA, en Proyecto UBACYT: La noción de ciudadanía sudamericana desde una perspectiva éticopolítica intercultural.

\section{INTRODUCCIÓN}

Paulo Freire es uno de los intelectuales latinoamericanos más importantes del siglo pasado que sigue vigente y siendo estudiado en este siglo.

$\mathrm{Su}$ obra teórica y su práctica político-pedagógica fueron intensa y extensa. Durante su largo recorrido como educador y militante enfrentó escenarios muy diversos que requirieron reposicionamientos teóricos y prácticos de gran intensidad, su obra muestra importantes giros y estratégicas tomas de posición. No es el mismo Freire quien trabaja con los círculos de cultura alfabetizando en el nordeste brasilero y que comienza a planificar un plan nacional de alfabetización, que el intelectual encarcelado y forzado al exilio que puesto en la necesidad de combatir la dictadura brasilera desde la diáspora. Tampoco es el mismo el Freire quien retorna luego de dieciocho años a un Brasil que ya se perfila mucho más neoliberal que genuinamente democrático o el que, pasado un tiempo, ejerce un cargo público en la Alcaldía de San Pablo ganada por primera vez por el Partido de los Trabajadores.

Y si bien no es el mismo desde sus posicionamientos teóricos y estrategias militantes, desde una perspectiva más profunda sigue siéndolo, sigue intacta su pasión por construir una educación al servicio de los desposeídos y por ver un Brasil más justo, democrático e igualitario.

En este artículo emprendo la tarea de rastrear en su producción teórica, alumbrada por su recorrido militante, los puntos nodales de su pensamiento que más allá de los posicionamientos estratégicos y las reelavoraciones teóricas relativas al contexto y a los escenarios en disputa, están presentes en toda su obra. Estos principios nos sirven hoy para pensar y repensar los desafíos de la educación en Latinoamérica y para postularlos como base de cualquier sistema educativo que busque construir una región más justa, libre, democrática y soberana.

A fin de alcanzar ese objetivo el trabajo comenzará planteando los puntos que hacen complejo el abordaje del pensamiento de Freire, luego propondrá una clasificación temporal y conceptual a fin de ordenar por etapas los distintos giros en la perspectiva del autor y finalmente identificará los puntos nodales que recorren todas las etapas y estructuran todos desarrollos arribando al núcleo de la reflexión freireana y a su legado más fecundo.

\section{COMPLEJIDAD DEL ABORDAJE DE LA OBRA Y PENSAMIENTO DE PAULO FREIRE}

La obra de Paulo Freire tiene muchas aristas que hacen complejo su abordaje. En primer lugar además del legado escrito encontramos una inmensa tarea práctica como pedagogo y organizador o partícipe de innumerables proyectos populares y políticos-pedagógicos. En segundo 
término encontramos la extensión de su producción: decenas de libros, ensayos, conferencias, seminarios en los que expone su pensamiento. Un tercer inconveniente es la asistematicidad y un marco teórico poco homogéneo en algunos de sus desarrollos pues sus reflexiones tienen una textura abierta, contextual y heterogénea, cita a K. Jaspers y a K. Marx, sin adherir ni al existencialismo ni al marxismo, solo por encontrar en ellos puntos interesantes. La reflexión de este pedagogo evoluciona, cambia a través del tiempo y de las profundas experiencias políticas y pedagógicas que marcaron su vida de educador e intelectual comprometido con la realidad en la que vivió. A veces la nueva forma que toman sus reflexiones parecen tener pocas relaciones y hasta contradecirse en algún punto, con las etapas anteriores.

A continuación describiré las dificultades que hacen complejo el abordaje del pensamiento freireano:

a) Relación entre la producción teórica y la práctica como educador militante: Si bien hace un esfuerzo enorme por ser coherente, su pensamiento evoluciona y se trasforma en la práctica, por lo que pueden encontrarse una serie de tensiones entre la obra escrita del educador y sus trabajos militantes en las distintas coyunturas y contextos histórico-sociales. La clave en este punto es entender que el pensamiento de un intelectual comprometido está profundamente vinculado con su acción por lo que evoluciona a la par de ésta. Freire es un intelectual pero, sobre todo, es un militante político, estas dos facetas se conjugan en su rol de pedagogo donde intenta unir reflexión y acción. Realiza un esfuerzo constante por ser coherente, por adecuar su acción a su reflexión y su reflexión a su acción, aún cuando sabe que es un esfuerzo trágico, que la coherencia total entre el discurso y la acción es un límite al que se puede intentar tender pero que difícilmente se puede alcanzar. Siguiendo sus propias enseñanzas, se puede afirmar que su obra escrita sirve para interpretar su acción, así como su acción para comprender lo que escribe.

b) Extensión de su obra: El segundo punto que trae dificultades es la extensión de la obra, que sería un problema menor si no se le sumara al tercer punto: el de la asistematicidad y el carácter abierto de su pensamiento, marcado por los avatares vitales y contextuales de su vida como pedagogo y militante. Esto genera un estilo oscuro, a veces confuso, poco sólido; cuando su pensamiento se dirige a lo concreto, a lo contextual, a la práctica educativa, se hace más claro e influyente. La extensión de su producción teórica presente en ensayos, artículos, conferencias, entrevistas, seminarios, etc, y la asistematicidad de la misma, hacen que su abordaje sea complicado, requiere un esfuerzo importante de interpretación, pero, finalmente pueden identificarse núcleos teóricos que recorren y articulan su obra. 
c) La asistematicidad, el carácter abierto de su pensamiento y un marco teórico poco homogéneo: Cuando uno aborda los ensayos del pedagogo, encuentra en muchos de sus textos una prosa oscura, una mezcla de temas y discursos, que en un primer momento confunden. Es más claro en textos que recuperan sus enseñanzas de conferencias, cursos, seminarios, entrevistas, cuando lo escrito refleja una práctica oral. Hay autores que sostienen que el contexto universitario en el que se desenvolvía lo "obliga" a teorizar, a fundamentar teóricamente su discurso, esta necesidad proveniente más de los requerimientos académicos del ámbito intelectual, que de su interés fundamental: los desarrollos necesarios para la acción. Extraer ideas claras sobre los temas que trata en sus ensayos requiere un esfuerzo considerable, los conceptos nunca están cerrados totalmente, a veces solo aparecen sugeridos. En sus conferencias, cursos, entrevistas, sus ideas suelen aparecer con mucha mayor nitidez. Podemos arribar a la siguiente conclusión: el interés primordial del educador es la práctica educativa, la reflexión sobre la práctica y la práctica a partir de la reflexión. La teoría por la teoría misma no tiene sentido, su pensamiento se hace más claro cuando logramos contextualizarlo y comprender que está íntimamente ligado a la acción. Por otra parte Freire hace una apología del diálogo, por lo que su pensamiento se desarrolla más cómodamente en situaciones donde prevalece la comunicación y el intercambio de ideas (entrevistas, conferencias, seminarios), que en los textos en donde el interés fundamental es la exposición y el desarrollo del pensamiento individual del autor.

La aparente ausencia de un marco teórico sólido está relacionada con la heterogeneidad de sus fuentes. Freire se nutre de varias vertientes intelectuales, cita a numerosos autores de la talla de: Marx, Hegel, Fromm, Bergson, Jung, entre una serie muy extensa. Sin embargo los interpreta de forma poco ortodoxa, solo por encontrar algún aspecto interesante en la reflexión de los citados y nunca adhiere dogmáticamente a sus doctrinas. La marca sólida y profunda que encontramos a lo largo de toda su reflexión es la del pensamiento cristiano tercermundista marcado por la impronta post conciliar: encíclica Mater et magistra, Concilio Vaticano II, encíclica Populorum progressius y los documentos de Medellín y Puebla del episcopado Latinoamericano. Es un cristiano militante que adhiere decididamente a la teología de la liberación.

Se reconoce como un intelectual que se mueve de una teoría a otra, de una corriente a otra sin demasiados reparos y, efectivamente, puede dar la impresión de que su reflexión carece de un marco teórico homogéneo. Sin embargo existe un matiz que está presente en toda su obra: el latinoamericanismo y el cristianismo tercermundista de su reflexión profundamente enraizado en sus prácticas como 
educador en el norte de Brasil primero y en otros terceros mundos luego se su exilio. La obra de Freire contiene ideas nuevas y revolucionarias que surgen en América Latina en los años 1960. Da cuenta de su formación católica el lenguaje imbuido de los conceptos liberacionistas provenientes de las corrientes progresistas del catolicismo de las que surge la teología de la liberación: pueblo, oprimidos, palabra, liberación, comunión, esperanza, amor. De acuerdo con Daniel Berisso (2012, p. 74), la afinidad entre los filósofos latinoamericanos provenientes de la llamada filosofía de la liberación y los teólogos tercermundistas es típica del clima intelectual en los que se el brasilero desarrolla parte fundamental de su obra. De esta vertiente brotará lo que consideramos el núcleo duro y el aporte original que Freire hace al pensamiento pedagógico y a la historia de la educación. Este núcleo duro se encuentra presente en toda la reflexión freireana y prevalece más allá de sus vicisitudes, de su fluidez y heterogeneidad.

El Paulo Freire de hoy tiene cierta coherencia
con el Paulo Freire de ayer. El Paulo Freire
de ayer no ha muerto. Quiero decir que yo
he estado vivo durante todos estos años [...].
Pero el Paulo Freire de hoy necesariamente
lleva consigo las marcas de la experiencia
[...]. Entonces, sería un desastre, sería muy
triste, si yo no hubiera aprendido con estos
cinco o seis momentos históricos, si hoy en
día yo siguiera siendo el mismo que llegó al
exilio veinte años atrás, primero en Bolivia y
después en Chile. Yo tengo aún que aprender
haciendo y viendo las cosas que se están
haciendo. (LEITE, 1979, p. 62).

La reflexión está marcada por la historia y las experiencias, se trasforma así como se trasforma el mundo en el que la reflexión acontece. Freire reclama su derecho (y hasta su deber) a repensar, reelaborar y reinventar su pensamiento. $\mathrm{Al}$ momento de interpretar su obra este es un punto clave a tener en cuenta: la reelaboración y la reinvención constante del pensamiento que intentamos abordar.

\section{ETAPAS DE LA REFLEXIÓN FREIREANA}

Teniendo en cuento lo dicho precedentemente en torno a la evolución del pensamiento del educador, a continuación distingo tres etapas ligadas estrechamente con los acontecimientos históricos y las experiencias personales que influyen en su reflexión:

a) Primera etapa: 1946-1964, comienza con sus primeras experiencias en educación de adultos y culmina con su debido a su desempeño en el Programa Nacional de Alfabetización del derrocado gobierno de J. Goulart. La obra más representativa de esta etapa 
es Educación como práctica de libertad. En este primer momento piensa todavía que la ciencia y la educación son relativamente neutrales, la relación política no es central (por lo menos explícitamente) en su forma de pensar la experiencia educativa. En esta etapa, sin embargo, desarrolla una tarea política pedagógica muy importante primero en el Servicio Social de la Industria de Recife (SESI) donde estaba a cargo del departamento de educación y, sobre todo, en el Movimiento de cultura popular (MCP) de Recife donde coordinaba los Círculos y Centros de Cultura y desarrolla y consolida su método de alfabetización. Esta práctica es importantísima, de ella surgirán los núcleos que conforman la médula de su pensamiento. Todavía no tematiza la politicidad de la educación pero la idea se encuentra en germen y será desarrollada en lo sucesivo.

El desarrollo teórico está puesto en la oposición naturaleza/cultura, hombre/animal. El objetivo de la educación es la liberación cultural: que el hombre tome conciencia de su condición de generador de cultura en tanto trabajador, alfabetizado o no, como medio para su desarrollo e instrumento para su liberación social.

b) Segunda etapa: Se corresponde con su exilio desde el año 1964 hasta su retorno definitivo a Brasil el 1980. Se hace manifiesta en Pedagogía del oprimido (FREIRE, 2005b). El cambio fundamental con respecto al primer momento es la explicita reivindicación del carácter político de la educación:

El Paulo Freire de ayer, un ayer que ubicaría
entre los años 50 y comienzos de los 6o, no
veía con claridad algo que el Paulo Freire
de hoy ve con mucha claridad. Y es lo que
denomino "la politicidad de la educación".
Esto es, la calidad que tiene la educación
de ser política. Porque la naturaleza de la
práctica educativa es política en sí misma, y
por eso no es posible hablar siquiera de una
dimensión política de la educación, pues toda
ella es política. Por lo mismo el Paulo Freire
de hoy - y a este lo ubico desde los fines de
los 6o y comienzos de los 70 - ve claramente
la cuestion de las clases sociales. (TORRES,
1986, p. 38).

El pensamiento del pedagogo brasilero aparecen categorías marxistas: clases, modos de producción, estructura y superestructura. Sin embargo, desde el punto de vista de este artículo, la forma en que plantea y trata la politicidad de la educación no encuentran su origen en el pensamiento de Marx, sino en la experiencia educativa inmediatamente anterior a su exilio. La raíz del pensamiento freireano está en el tercemundimo o latinoamericanismo de su experiencia y en su carácter de cristiano radical militante, las categorías marxistas tienen 
un papel auxiliar. La única categoría de importancia que proviene de esta vertiente es el tratamiento de la relación opresor-oprimido en la matriz de la dialéctica hegeliana del señor y el siervo, pero aún esta categoría es incorporada de una manera poco ortodoxa.

Esta etapa de radicalización de su pensamiento coincide con su exilio y su experiencia educativa en otros países latinoamericanos y en otros sectores del tercer mundo. De esta experiencia también surge otra de sus obras paradigmáticas Cartas a Guinea Bissau. Adriana Puiggrós (1998) sostiene que la cárcel y el exilio al que fue sometido por el golpe militar llevan al educador brasilero hacia el discurso teórico de la izquierda más ortodoxa, alejándolo de los desarrollos más ligados con una izquierda con un perfil latinoamericano.

Según la nueva visión existe un tipo de educación (bancaria) que sirve para consolidar la dominación y el sometimiento de los sectores oprimidos por los opresores de las sociedades capitalistas. En oposición a ella existe otra forma de ejercer y pensar la educación (dialógica) que será un instrumento importantísimo para la lucha por la liberación de los oprimidos. La práctica freireana y su método de alfabetización se colocan en la primera línea de acción y se trasforman en un instrumento para las clases oprimidas en su lucha por cambiar las estructuras sociales que las mantiene sometidas.

El giro en su pensamiento se hace visible en las citas bibliográficas y referencias teóricas, en la primer etapa suele citar a M. Scheler, J. Ortega y Gasset, K. Mannhein, A. Whiteheat, etc.; en la segunda etapa a Marx, Lenin, Mao, H. Marcuse, F. Fanon etc.

c) Tercera etapa: La última etapa, 1980-1996 está ligada a su retorno al Brasil luego de años de exilio y a la aparición de un nuevo y poderoso enemigo a enfrentar: el neoliberalismo.

Se caracteriza por la relectura de las obras del segundo período a fin de adecuar la reflexión y la práctica educativa a la lucha en el nuevo contexto en el que la revolución y el cambio radical de las estructuras sociales habían dejado de ser una posibilidad más o menos inmediata. En cambio, el poder hegemónico del discurso neoliberal extendía su presencia y poder a todos los terrenos de disputa.

Los libros más representativos de esta etapa son Pedagogía de la esperanza y Pedagogía de la autonomía - saberes necesarios a la practica esperanza (FREIRE, 2005a, 2008). La escuela dejó de ser parte de una estructura de opresión que solo sería modificable por un proceso revolucionario alimentado desde espacios educativos informales como los Círculos de Cultura. En el nuevo contexto el aula se trasforma en un territorio de lucha frente al neoliberalismo que trata de convertirla en un espacio rentable económicamente, a riego de hacerlo desaparecer, para la formación de individuos en competencias relevantes para su desempeño en el mercado 
1 Durante esta etapa también se hace evidente la influencia del el contexto sociohistórico sobre su pensamiento, ya que interviene en la discusión modernidadpostmodernidad reelaborando categorías, resignificando conceptos, haciendo constantes autocríticas y reinvenciones de su pensamiento: "Existe una postmodernidad en la derecha, pero también existe una postmodernidad de izquierda y no, - como casi siempre se insinúa -, cuando no se insiste, que la postmodernidad es un tiempo demasiado especial que suprimió clases sociales, ideologías, izquierdas y derechas, sueños y utopías. Y uno de los aspectos fundamentales para la postmodernidad de izquierda es el tema del poder, el tema de su reinvención que trasciende el de la modernidad, el de su pura conquista" (FREIRE, 1996, p. 189).
2 Así en el prólogo a un libro de Moacir Gadotti señala "Gadotti lo construye, lo sitúa, a mi juicio, como un pensador progresivamente posmoderno. Un pensador que sabe que, para obtener verdades, es necesario no estar demasiado seguro de ellas. No hay certeza, a no ser de la incerteza de lo que parece absolutamente cierto. "[...] Una de las notas positivas que caracterizan este libro es que siendo un texto osado, 'poseedor de voluntad', dueño de cierta cara, afirmado en una cierta posición, no transpira, sin embargo, arrogancia. No sugiere siquiera que la suya es la única cara, que fuera de su verdad no hay solución. Una vez más, su postmodernismo. Lo que el texto deja entre líneas es la esperanza de su autor de que sus lectores y lectoras se asuman como productores de la comprensión de su texto, en vez que simplemente la busquen como algo que él hubiese dejado para ser descubierto por ellos y ellas" (FREIRE, 1996). laboral. La escuela y educación formal, se vuelven terreno de disputa política, lugares que reclaman ser reinventados como instrumentos para la libertad y espacios de resistencia frente a la hegemonía neoliberal ${ }^{1}$.

Durante esta etapa Freire se desempeña como Secretario Municipal de Educación de la Intendencia de San Pablo frente a la cual estaba la alcaldesa Luiza Erudina, referente del Partido de los Trabajadores (PT), donde intentó reconstruir y reinventar la red pública de escolaridad.

Recapitulando, la enorme tarea práctica, la pasión militante y el esfuerzo constante por la coherencia entre la reflexión y la acción deben ser tomados en cuenta seriamente a la hora de interpretar la producción teórica de Paulo Freire, así como su obra teórica es vital para interpretar su acción.

Es cierto que la extensión de la obra combinada con la asistematicidad, la aparente ausencia de un marco teórico homogéneo y la variedad un tanto ecléctica de su fuentes y citas, hacen difícil el abordaje de la misma. Sin embargo tenemos la seguridad de que hay una serie de convicciones teóricas que guían la reflexión y la práctica constituyendo la médula de su pensamiento, en ellas encontraremos las claves para interpretarlo. Justamente uno de estos núcleos tiene que ver con el derecho al cambio; esta es una facultad que reivindica como propia. No hay tema, idea o palabra que no se pueda someter al diálogo, a la discusión abierta, al intercambio, a la mutación y a la reinvención. El derecho y el deber de reinventarse se vuelven notábles en el último período. ${ }^{2}$

A continuación seguiremos en la aventura de rastrear y exponer los ejes en la reflexión del fecundo pedagogo.

\section{EL NÚCLEO DE SU PENSAMIENTO}

Más allá de las dificultades y lo arduo que resulta interpretar la obra del autor, puedo identificar tres cuestiones fundamentales que dan solidez a su pensamiento y son al mismo tiempo el núcleo duro de la reflexión freireana, la clave de interpretación de su obra y el aporte más original que el brasilero hace a la pedagogía:

a) La afirmación del carácter político de la práctica educativa. La educación así como la producción y reproducción del conocimiento no es una práctica neutra, es una actividad política. La politicidad de la educación se manifiesta en varios sentidos y en varios niveles. En el proceso educativo existen relaciones de poder que pueden entramarse de distinta forma para conformar espacios opresivos y de dominación o espacios de libertad y liberación.

b) Dialogicidad: Si queremos que la educación sea un espacio para la libertad debe ser una actividad dialógica, donde los educadores sean a la vez educandos y los educandos educadores, a fin de 
crear una relación de intercambio y comunicación donde construyan y recreen conocimiento. Esta forma se debe oponer a la tradicional forma de trasmitir conocimientos que llama "bancaria" donde el educador trasfiere o deposita conocimiento en el educando que es una especie de receptáculo vacío y pasivo del saber del maestro. La educación dialógica implica entramados de poder en el ámbito de la educación que estimulan las prácticas liberadoras y desactivan las opresivas.

c) Partir del contexto del educando: El proceso educativo de corte liberador y dialógico debe estar centrado en el contexto del alumno, en el entorno en el que se ha desarrollado, pues no es posible aprender si el conocimiento está en contradicción con la vivencia personal de quien aprende. El maestro debe partir de este entorno, debe aprenderlo; éste es el primer paso de la relación pedagógica. Al final del proceso educativo educador y educando vuelven a repensar su entorno con los nuevos conocimientos para comprender mejor su propia realidad.

Estos tres elementos, que actualmente forman parte de muchos discursos pedagógicos de corte progresista, en su momento fueron revolucionarios y lograron ser articulados en un método de alfabetización que resultó un instrumento formidable en la tarea pedagógica realizada por Freire en el Brasil. Este método, así como los principios que lo inspiran, ha sido retomado como modelo por infinidad de grupos de educadores populares que desarrollan su actividad político-pedagógica en los sectores más olvidados de Latinoamérica y el Tercer Mundo.

La importancia de estos nodos, además de su carácter de principios teóricos que deben regir el proceso educativo, reside en la posibilidad de articular los mismos en un método alfabetización y post alfabetización con aplicaciones y resultados asombrosos.

A continuación desarrollaremos en forma más extensa los ejes del pensamiento enunciados arriba, a fin de introducirnos en la médula de los mismos.

\subsection{La politicidad de la educación}

La educación definitivamente es una práctica política, en ella se encuentra involucrado el poder en distintos niveles y de distintas formas; no es neutral, implica, siempre, tomar partido.

La práctica educativa no solo tiene aspectos o matices políticos es política; esta característica se manifiesta fundamentalmente en dos niveles:

a) la politicidad de la relación educador-educando. Existe una relación política en el vínculo básico de la práctica educativa: el que relaciona al que enseña y al que aprende.

b) El otro aspecto político de la educación tiene que ver 
con su contenido, con lo que se enseña, podemos llamarlo contenido programático de la educación.

\subsubsection{La relación política educador-educando}

¿Quién conoce en la práctica educativa? Al hacer esta pregunta creo que empezamos a percibir que su contestación no es exclusiva del ámbito de la pedagogía. [...] Cuando hacemos esta primera pregunta, parece que hay algo que empieza por lo menos, a ser matizado por la política. Empezamos a ver que hay formas diferentes de contestar esta pregunta. Y estas mismas conllevan el primer cuerpo de la respuesta, una cierta marca ideológica y una cierta opción política. [...] Por ejemplo, [...] si uno contesta que quien sabe en la práctica educativa es el profesor, uno ha de preguntar enseguida: entonces ¿cuál es el papel de alumno? Quien dice que en la práctica educativa es el profesor quien sabe, dirá también para ser coherente, que el papel del alumno es aprender. Entendiendo el acto de aprender como el de recibir conocimiento del profesor. El profesor enseña y el alumno estudia y aprende. (FREIRE, 1988).

Así como Foucault percibe claramente que el componente de poder que existe en las relaciones educativas se vincula íntimamente con la posesión del saber (FOUCAULT, 2000), Freire entiende perfectamente que en las relaciones maestro- alumno hay relaciones de poder establecidas desde un discurso que determina quien tiene saber y quien no (y por lo tanto quien tiene poder y quien no). La posesión del saber determina las relaciones y los roles entre los participantes en la práctica educativa.

Esta intuición de la politicidad intrínseca de la relación educativa recorre todo el pensamiento de Freire pero tiene su expresión más enfática en la Pedagogía del Oprimido. En este texto aparecen claramente conceptos provenientes de sus lecturas de Marx, Lenin, Mao, E. Guevara, L. Althusser, G. Lukács, Marcuse, Fanon: clase social, lucha de clases, estructura, superestructura, revolución, partido, opresor, oprimido, alienación etc. Eran momentos en que se vislumbraba una revolución que cambiaría las estructuras sociales injustas de los países latinoamericanos. Freire estaba en el exilio luego de ser encarcelado y expulsado de su país por el golpe del estado del año 1964.

Durante este período la relación pedagógica educador-educando tal como se daba en el contexto brasilero y en la mayoría de las sociedades modernas, es traducida en términos de opresor-oprimido. La educación "bancaria", donde el alumno ocupa el lugar de depósito del saber del profesor no hace más que consolidar la estructura de explotación y de opresión imperante. Este tipo de educación consolida el lugar de oprimido que ocupa 
el alumno proveniente de sectores populares: el que no sabe, el ignorante, el que tiene que obedecer al que posee el saber (el opresor): en la escuela al maestro, en la fábrica al dueño, en el campo al capataz, en la calle al policía. Freire propuso una pedagogía que revolucionará la forma en que circulaba el poder y el saber, una pedagogía que liberara tanto al opresor como al oprimido. El instrumento fundamental será el diálogo y el punto de partida el contexto sociocultural y las experiencias previas del alumno. La tesis que sostiene en esta etapa afirma que para que exista un real y definitivo cambio en el proceso educativo que permita implementar masivamente la educación para la libertad, debían transformarse las estructuras sociales injustas. Hasta tanto no se realice una revolución en este ámbito, la pedagogía del oprimido tendría la función de preparar el terreno para el cambio social desde los espacios educativos no formales, pero su difusión hacia todos los ámbitos de la sociedad no sería posible hasta que no se genere un cambio revolucionario (FREIRE, 2005b).

En la sucesiva evolución de su pensamiento, a la par que desaparece el contexto setentista en que la revolución social parecía inminente, las categorías tan fuertemente marcadas en esta etapa se atenúan, deja de utilizar tan enfáticamente los términos opresor - oprimido para referirse a la relación educador - educando, y también matiza la necesidad de un cambio revolucionario previo para que sea posible una educación liberadora. Sin embargo subsiste siempre en el centro de su reflexión la idea de que la educación es una práctica política, que dentro de ella se dan relaciones de poder y de saber, de opresión o de liberación. Esta afirmación está en la base, en la matriz de su pensamiento y es guía de su acción dirigida a la creación y/o trasformación de los ámbitos educativos en territorios donde la opresión se encuentre ausente.

\subsubsection{El contenido del conocimiento que se trasmite}

No basta saber leer que "Eva vio una uva", es necesario saber qué posición ocupa Eva en el contexto social, quién trabaja en la producción de la uva y quién lucra con este trabajo. No solo la relación pedagógica maestro-alumno es de naturaleza política. Otro aspecto claramente político en el proceso educativo es el tipo de conocimiento que se trasmite. El saber genera poder y el poder saber. Trasmitir saberes vacíos, aparentemente neutros ("mi mamá me mima" o "mi mamá me ama"), aislados de la realidad que le toca vivir al alumno y al educador, además de ser inútiles para comprender esa realidad, son un obstáculo para esa comprensión. Es fundamental, para trasformar las relaciones de poder dentro del proceso educativo, modificar el tipo de saber que se trasmite: lo que se enseña tiene que ser significativo para quien aprende, el saber debe estar enraizado en la experiencia previa del alumno y no aislado de esta. 


\subsection{La educación dialógica}

El diálogo como instrumento de enseñanza tiene una tradición antigua, se remonta, por lo menos, a la mayéutica socrática. Para Freire es el instrumento central del un proceso de aprendizaje, sin él no hay educación verdadera. El freireano tiene una función adicional al diálogo socrático (en la que el hábil maestro sacaba del alumno verdades que, por ser eternas, ya estaban en él); además de la función pedagógica tiene una función política: modificar las relaciones de poder, democratizar el ámbito de la enseñanza, hacer que el poder y el saber circulen de forma diferente.

El método socrático partía de la ironía: el maestro sostenía su propiaignorancia sobrealgún tema einterrogaba a quien supuestamente poseía este conocimiento. En el diálogo, Sócrates, dejaba en evidencia que el alumno no sabía lo que creía saber, el diálogo seguía transcurriendo y el maestro guiaba al alumno hacia alguna idea o concepto sobre el que había meditado durante mucho tiempo, finalmente el discípulo no tenía más remedio que aceptar estos conceptos.

La educación dialógica, tal como la comprende el brasilero, requiere otras condiciones para desarrollarse: horizontalidad, confianza, esperanza.

Poner el intercambio horizontal y la confianza en el centro del proceso de enseñanza implica modificar la forma en que tradicionalmente circula el poder y el saber en estos ámbitos. El diálogo no parte de una posición irónica, al contrario, requiere complicidad entre los que intervienen. Tampoco la estrategia consiste en demostrar que maestro o discípulo son ignorantes; se parte de la concepción de que todos los que intervienen tiene conocimientos valiosos para aportar al diálogo, así como todos son parcialmente ignorantes, en el sentido de que hay cosas que no saben, por lo que todos pueden aprender.

El educador tiene saberes, conocimientos valiosos para trasmitir, pero también los tiene el alumno. Reconocer esto es básico; si el alumno no tiene nada que decir o aportar, el diálogo se trasforma en monólogo. El docente, que ocupa una posición de mayor responsabilidad dada su preparación, debe partir del contexto del alumno, de sus experiencias, sus saberes previos y así establecer una relación que tenga significado, contenido concreto y comprensible de modo que permita una efectiva comunicación y trasmisión de conocimientos.

Esta apelación al diálogo puede parecer ingenua, trillada, poco original, sin embargo veremos que, si se quiere establecer realmente un método de enseñanza que lo tenga como centro, deben transformarse los sujetos y el objeto de la enseñanza tradicional. En otras palabras poner el diálogo verdadero en el centro del proceso de formación implica deconstruir el papel tradicional del docente y del alumno, relativizarlos, pero también, 
3 I. OBTENCIÓN DEL UNIVERSO VOCABULAR DE LOS GRUPOS CON LOS CUALES SE TRABAJARÁ. "Este estudio se hace a través de encuentros informales con los moradores del área a alfabetizar y durante los cuales no solo se obtendrán los vocablos con sentido existencial, y por tanto de mayor contenido emocional, sino también aquellos típicos del pueblo, sus expresiones particulares, vocablos ligados a la experiencia de los grupos [...]. De esta fase se obtiene resultados muy ricos para el equipo de educadores no solo por las relaciones que se establecen, sino por la riqueza del lenguaje del pueblo, que a veces ni se sospechan. La entrevistas revelan deseos, frustraciones, descreimientos, esperanzas, deseos de participación, como también momento altamente estéticos en el lenguaje del pueblo [...]. Las palabras generadoras deberían salir de este estudio y no de una selección hecha por nosotros en nuestro gabinete, por mas técnicamente bien escogidas que estuviesen" (FREIRE, 1969, p. 109). trasformar la concepción de cómo se produce (y no solo como se trasmite) el conocimiento: implica una trasformar el proceso educativo desde sus bases.

\subsection{Partir del contexto de los educandos}

Los puntos que marcamos como núcleos de la reflexión freireanos están estrechamente relacionados entre sí. Existe una relación de implicación del primero al segundo y de este al tercero, así la coherencia del método se hace evidente.

La educación es una actividad eminentemente política: hay relaciones de poder y de saber. El proceso educativo puede tomar características opresivas o liberadoras; esto depende de la opción que tomen educadores $\mathrm{y} / \mathrm{o}$ educandos. Si tomamos la segunda opción debemos aceptar que el sistema educativo tiende a reproducir la opresión imperante en el sistema social; la educación "bancaria" no hace más que consolidar las relaciones de poder existentes y el sistema de opresión. Por lo tanto necesita ser modificada, trasformada, reemplazada por un modelo pedagógico que permita la liberación de los oprimidos (y la de los opresores); este modelo tendrá como eje el segundo núcleo de pensamiento. La educación debe ser un proceso dialógico, en que el poder y el saber circulen de manera horizontal, democrática, en las que todos sean educadores y educandos alternativamente. Para que el diálogo sea posible, para que no sea una mera enunciación formal sino un hecho real de comunicación, intercambio, creación y recreación de conocimientos debemos partir del universo de significado del alumno, de su lenguaje, surgidos de su experiencia cotidiana, de otra forma la trasformación del proceso y el diálogo mismo no serán más que una ficción.

¿Qué quiere decir: "partir del contexto del educando"? Significa familiarizarse con los vocablos con sentido existencial, emocional, con las expresiones particulares ligadas a las experiencia del (o de los) que aprende(n). Y a través de ellos comprender los deseos, frustraciones, descreimientos y esperanzas que los movilizan ${ }^{3}$. De este momento previo surgen, en el "método Freire", las "palabras generadoras".

Las palabras generadoras, vocablos con los que se comienza a alfabetizar, tienen que ser significativas para los educandos. Se aprende a leer desde lo ya comprendido, desde palabras que tiene plena significación para quienes trabajan con ella, que son familiares, se refieren a experiencias cotidianas, a sus deseos, frustraciones y esperanzas. Es mucho más fácil incorporar el lenguaje escrito a través de palabras conocidas que tienen pleno sentido en el lenguaje oral y en la experiencia cotidiana, en vez de empezar con frases de poca significación o directamente sin sentido para los educandos. Se incorpora la cultura letrada a partir de la cultura oral, de modo que lo 
4 Toda lectura de la palabra presupone una lectura anterior del mundo, y toda lectura de de la palabra implica volver sobre la lectura del mundo, de manera tal que leer mundo y leer palabra se constituyen en un movimiento donde no hay ruptura, en donde uno va y viene. Y leer mundo junto con leer palabra en el fondo, para mi significan reescribir el mundo. Reescribir con comillas, quiere decir trasformarlo. La lectura de la palabra debe estar inserta en la comprensión de la transformación del mundo, que provoca la lectura de él y debe remitirnos siempre de nuevo a la lectura del mundo (FREIRE, 1988, p. 78). aprendido se inserte y adquiera significación en su universo; de esta manera, el alumno dice "su" palabra. Cuando se incorpora la cultura letrada al mundo del alumno, adquiere un nuevo instrumento que le permite acceder a saberes que le estaban vedados o que, en su contexto, no tenían sentido. De este modo se va ampliando su capacidad de comprensión y su universo significativo.

El esquema recoge una intuición fundamental que está en la base del método freireano y de toda su pedagogía: el conocimiento se adquiere y se asimila, se construye y se trasforma, yendo de lo conocido a lo desconocido y viceversa. Lo desconocido toma significado a partir de lo que se conoce. A su vez lo ya conocido también es re-significado, dando como resultado un conocimiento más complejo donde se encuentra presente el conocimiento viejo re-significado por el nuevo y el nuevo conocimiento incorporado y resignificado por el viejo. En algo similar a la idea de círculo hermenéutico pero en el plano del aprendizaje. ${ }^{4}$

Esta idea es fundamental no solo para la alfabetización o postalfabetización, es aplicable a todo proceso pedagógico, a la enseñanza en todos sus niveles.

Freire sostiene, con razón, que tradicionalmente el sistema educativo trasmite el saber, el lenguaje, los significados, la visión y en definitiva la cultura de la clase o el grupo que tiene el poder (es decir tiene el poder y el saber). Por eso los que pertenecen a otras culturas o subculturas y tiene otras experiencias, suelen fracasar mucho más fácilmente; y si no fracasan, son incorporados dócilmente al sistema de dominación. Reconociendo el saber previo del educando, su cultura o subcultura, su universo lingüístico y vivencial, se rompe con el esquema opresivo que "congela" el círculo hermenéutico. Lo congela porque considera verdadero, válido y real el saber de los que tiene el poder, despreciando, rebajando y, si es posible, anulando o destruyendo saberes diferentes, experiencias y universos lingüísticos y culturales distintos. El método Freire pone a funcionar nuevamente este círculo.

\section{CONCLUSIÓN}

Cumplimos el objetivo del artículo. A partir de los tres puntos nodales identificados (politicidad, dialogicidad y contexto del educando) Freire sienta las bases de un esquema pedagógico para la libertad. Podemos sintetizar lo dicho del siguiente modo:

En primer lugar debemos reconocer el carácter eminentemente político de la educación. Que el proceso educativo sea liberador u opresor, dependerá de la opción que estemos dispuestos a tomar. Si tomamos la postura que considera a la educación como una actividad neutra, que tiene como finalidad trasmitir conocimientos objetivos, verdaderos y enseñar a usar correctamente la razón, sin advertir que tras este discurso suele ocultarse un esquema pedagógico que refuerza las relaciones de opresión y 
sometimiento vigentes en el sistema social, el proceso educativo será un mecanismo más de opresión.

En cambio si reconocemos que tras el esquema pedagógico presente se ocultan mecanismos de disciplinamiento, técnicas de obturación de los procesos de compresión y conocimiento con el efecto de consolidar un sistema de dominación y de reproducción acrítica de valores y saberes, entonces debemos trabajar a favor de un sistema educativo distinto. Con este reconocimiento se da el primer paso en la construcción de una pedagogía para la libertad y la liberación.

El paso siguiente es deconstruir el lugar del docente como sujeto único en el proceso educativo, que posee el saber completo, que tiene la autoridad, la palabra, el que actúa, frente a la pasividad del educando que es una especie de receptor vacío a ser llenado por el conocimiento transmitido. Para esto debemos reconocer que el alumno posee saberes previos, experiencia, que comprende el mundo pues efectivamente vive en él. Por lo tanto, para incorporar conocimientos nuevos, precisa que estos sean significativos, que tengan contacto con su universo simbólico. Así se sientan las bases para la dialogicidad de la educación. El docente debe partir de lo que saben los alumnos, debe comenzar aprendiendo de ellos, para luego trasmitir su conocimiento. Recíprocamente los alumnos darán una visión del saber del maestro que, seguramente, le servirá a este para complejizar y comprender mejor su propio saber.

Es decir partimos de una pedagogía en donde todos son educadores y educandos, en donde todos enseñan y aprenden, en donde nadie tiene el conocimiento absoluto, el saber verdadero, donde se ponen en diálogo formas diferentes de ser y estar en el mundo, formas distintas de comprenderlo. En este esquema el poder y el saber circulan democráticamente a través del diálogo, sin quedar concentrados en ninguno de sus polos.

En este entramado democrático de poder-saber se pueden crear subjetividades libres, pues tanto el alumno como el maestro pueden canalizar su impulso de ser más, pueden comprender que no deben quedarse encerrados en los roles que se les atribuyen desde el discurso dominante (el del trabajador analfabeto, el de campesino ignorante, el de oficinista mediocre, o el de burócrata sin perspectivas) sino que pueden transitar hacia otros formas de ser persona convertirse en militantes políticos, campesinos que cuidan de la naturaleza pues la conocen y la aman, trabajadores conscientes de sus derechos, artistas, intelectuales, o lo que fuera. Una educación para la libertad no define de una vez y para siempre a los que intervienen en ella, no los limita a una sola forma de ser y saber, a una sola perspectiva, al contrario canaliza el deseo de todo ser humano de transformarse, de ser más, de evolucionar hacia otras formas de ser persona.

Y sobre todas las cosas, una educación para la libertad, permite que cada uno diga su palabra y dé una 
interpretación original de sí mismo y de lo que lo rodea. No es la autoridad del docente, del jefe o del dueño de la tierra, la que tiene poder de decir quién soy, como es el mundo a mi alrededor y que debo decir de él. La educación para la libertad nos hace dueños de nuestras palabras, ese es su objetivo más profundo.

\section{REFERÊNCIAS}

BERISSO, Daniel. Los límites del concepto de ciudadanía en el marco de una ética latinoamericana (De una ética de la liberación a una praxis intercultural). 2012. oo f. Tesis (Doctorado en Filosofia) - Universidad de Buenos Aires, Buenos Aires, 2012.

FREIRE, Paulo. La educación como práctica de la libertad. Traducción Lilian Ronzoni. Montevideo: Ed. Siglo Veintiuno, 1969.

FREIRE, Paulo. La educación para una transformación radical de la sociedad. In: MAZÓ, Pere Soler (Org.). Una educación para el desarrollo: la animación sociocultural. Madrid: Fundación Banco Exterior, 1988. p. 13-24.

FREIRE, Paulo. Prólogo. GADOTTI, Moacir. Pedagogía de la praxis. Buenos Aires: ed. Miño y Dávila, 1996.

FREIRE, Paulo. Pedagogía de la Esperanza. México: Siglo XXI Editores, 2005a.

FREIRE, Paulo. Pedagogía del oprimido. 2. ed. México: Siglo XXI Editores, 2005b.

FREIRE, Paulo. Pedagogía de la autonomía: saberes necesarios a la practica esperanza. 2. ed. Buenos Aires: Siglo XXI Editores, 2008.

FOUCAULT, Michel. Vigilar y Castigar. México: Ed. Siglo Veintiuno, 2000.

LEITE, Ligia Chiappini Moraes. Encontro com Paulo Freire. Educação \& Sociedade, San Pablo, Campinas, v. 1, n. 3, p. 47-75, maio 1979.

PUIGGRÓS, Adriana. Prólogo. In: FREIRE, Paulo; BETTO, Frei. Esa escuela llamada vida. Tradução de Estela Dos Santos. Buenos Aires: Legasa, 1988. p. 7-63.

TORRES, Rosa María. Educación Popular: un encuentro con Paulo Freire. Quito: CECCA-CEDECO, 1986. 